\title{
On Smoothing Problems with One Additional Equality Condition*
}

\author{
S. Asmuss ${ }^{1}$ and N. Budkina ${ }^{2}$ \\ ${ }^{1}$ University of Latvia \\ ${ }^{2}$ Riga Technical University \\ ${ }^{1}$ Zellu 8, LV-1002, Riga, Latvia \\ ${ }^{2}$ Meza iela 1/4, LV-1048, Riga, Latvia \\ E-mail(corresp.): budkinanat@navigator.lv \\ E-mail: asmuss@lanet.lv
}

Received September 15, 2007; revised July 23, 2008; published online April 30, 2009

\begin{abstract}
Two problems of approximation in Hilbert spaces are considered with one additional equality condition: the smoothing problem with a weight and the smoothing problem with an obstacle. This condition is a generalization of the equality, which appears in the problem of approximation of a histogram in a natural way. We characterize the solutions of these smoothing problems and investigate the connection between them.
\end{abstract}

Key words: Smoothing problem, approximation, spline.

\section{Formulation of Problem of Smoothing Histopolation}

The idea to use splines for solving the problem of approximation of a histogram firstly appeared in the paper of Boneva, Kendall and Stefanov ([2]). Schoenberg investigated the solution of such problems in context of the theory of splines ([10]). During the last three decades the problem of approximation of the density function of a random value by splines has been investigated in various statements and under different restrictions on its solutions.

The problem of nonparametric estimation of a density function and its formulation is still being topical, this fact is confirmed for example by papers $[1,3,6,7,12,13]$. Firstly for the solution of this problem interpolation splines were used, later smoothing splines were considered to be more useful. In the present paper smoothing splines are investigated under one additional condition, which we consider to be very important, as it corresponds to the property of density histogram (the area under it is equal to 1 ). We consider the problem of approximation of a density histogram in two different formulations: as a

\footnotetext{
* This study was partially supported by The European Social Fund (ESF)
} 
problem of smoothing histopolation with a weight parameter and as a problem of smoothing histopolation with an obstacle, we obtain the connection between their solutions. This connection allows us to reduce the problem with an obstacle to the problem with a weight parameter, and using the algorithm for solving the easier problem to find the solution of a problem with an obstacle. The method of reducing one problem to another has been effectively used for smoothing problems without additional restrictions on their solutions (see, e.g. [8]).

Let $\triangle_{n}=\left\{a=t_{0}<t_{1}<\ldots<t_{n}=b\right\}$ be a mesh on the interval $[a, b]$, and let $F=\left\{f_{1}, \ldots, f_{n}\right\}$ be a corresponding density histogram, i.e. $f_{i}$ is the frequency for the interval $\left[t_{i-1}, t_{i}\right]$, where $i=1, \ldots, n$. The mesh steps are denoted by $h_{i}=t_{i}-t_{i-1}, i=1, \ldots, n$.

In many practical applications it is of interest to have a function $g$ that satisfies the area matching histopolation conditions

$$
\int_{t_{i-1}}^{t_{i}} g(t) d t=f_{i} h_{i}, \quad i=1, \ldots, n .
$$

(see, for example, $[4,5]$ ).

The problem of histopolation is solvable, but not uniquely. We suggest to use the following smoothing functional

$$
\int_{a}^{b}\left(g^{(q)}(t)\right)^{2} d t
$$

as an objective function. Then we get a histopolation problem in the Sobolev space $\mathbf{W}_{2}^{q}[a, b]$ :

Problem $1^{0}$ (histopolation problem) Find $g$ such, that

$$
\begin{aligned}
& \int_{a}^{b}\left(g^{(q)}(t)\right)^{2} d t \longrightarrow \quad \min _{g \in \mathbf{W}_{2}^{q}[a, b],} \\
& \int_{t_{i-1}}^{t_{i}} g(t) d t=f_{i} h_{i}, \quad i=1, \ldots, n \text {. }
\end{aligned}
$$

Taking into account that the information on frequencies $f_{i}, i=1, \ldots, n$ is obtained in practice as a result of measuring, experiments or preliminary calculations, it may be inexact. Thus we consider a more general case of the problem of approximation of histogram. Depending on the known information about the frequencies, the problem of smoothing histopolation can be considered with different types of restriction on the solution. Let us consider two problems of smoothing histopolation for given parameters $\omega>0$, delta $>0$.

Problem $2^{0}$ (the smoothing histopolation with a weight parameter $\omega$ ). Find $g$ such, that

$$
\begin{aligned}
& \int_{a}^{b}\left(g^{(q)}(t)\right)^{2} d t+\frac{1}{\omega} \sum_{i=1}^{n}\left(\int_{t_{i-1}}^{t_{i}} g(t) d t-f_{i} h_{i}\right)^{2} \longrightarrow \quad \min _{g \in \mathbf{W}_{2}^{q}[a, b],} \\
& \int_{a}^{b} g(t) d t=1 \text {. }
\end{aligned}
$$


Problem $3^{0}$ (the smoothing histopolation with an obstacle) Find $g$ such, that

$$
\begin{gathered}
\int_{a}^{b}\left(g^{(q)}(t)\right)^{2} d t \longrightarrow \\
g \in \mathbf{W}_{2}^{q}[a, b] \\
\sum_{i=1}^{n}\left(\int_{t_{i-1}}^{t_{i}} g(t) d t-f_{i} h_{i}\right)^{2} \leq \delta, \int_{a}^{b} g(t) d t=1 .
\end{gathered}
$$

In the case of exact information the last condition $\int_{a}^{b} g(t) d t=1$, describing the conservation property of density histogram, holds unconditionally. But in the case of inexact information this requirement is essential.

We investigate these two problems, prove the existence of their solutions and show the connection between them in a more general case.

\section{A Generalization of the Problem of Smoothing Histo- polation}

Let $X, Y$ be Hilbert spaces and assume that a linear operator $T: X \rightarrow Y$ and linear functionals $k_{i}: X \rightarrow \mathbb{R}, i=1, \ldots, n$, are continuous, $T(X)=Y$, $\operatorname{dim} \operatorname{Ker} T<+\infty$. Here and in the sequel $\operatorname{Ker} T$ is the kernel of the operator $T$. We define the operator $A=\left(k_{1}, \ldots, k_{n}\right)$ and suppose that $\operatorname{Ker} T \cap \operatorname{Ker} A=\{0\}$ and $A(X)=\mathbb{R}^{n}$.

For a given vector $\boldsymbol{r}=\left(r_{1}, \ldots, r_{n}\right)$ and parameters $\delta, \omega>0$ we consider the following minimization problems:

Problem 1 (the interpolation problem)

$$
\min \left\{\|T g\|^{2}: \quad k_{i} g=r_{i}, \quad i=1, \ldots, n, \quad g \in X\right\},
$$

Problem 2 (the smoothing problem with a weight)

$$
\min \left\{\|T g\|^{2}+\frac{1}{\omega}\|A g-\boldsymbol{r}\|^{2}: \sum_{i=1}^{n} k_{i} g=\sum_{i=1}^{n} r_{i}, \quad g \in X\right\},
$$

Problem 3 (the smoothing problem with an obstacle)

$$
\min \left\{\|T g\|: \sum_{i=1}^{n}\left(k_{i} g-r_{i}\right)^{2} \leq \delta, \quad \sum_{i=1}^{n} k_{i} g=\sum_{i=1}^{n} r_{i}, \quad g \in X\right\} .
$$

It is easy to see that Problem $1^{0}$, Problem $2^{0}$ and Problem $3^{0}$ are a particular cases of Problem 1, Problem 2 and Problem 3 respectively, if

1. $X=\mathbf{W}_{2}^{q}[a, b], Y=\mathbf{L}_{2}[a, b]$,

2. the operator $T: \mathbf{W}_{2}^{q}[a, b] \rightarrow \mathbf{L}_{2}[a, b]$ is the operator of differentiation $T g=g^{(q)}$, 
3. $k_{i} g=\int_{t_{i-1}}^{t_{i}} g(t) d t, i=1, \ldots, n, A=\left(k_{1}, \ldots, k_{n}\right)$,

4. $\boldsymbol{r}=\left(r_{1}, \ldots, r_{n}\right), \sum_{i=1}^{n} r_{i}=1$, where $r_{i}=f_{i} h_{i}, i=1, \ldots, n$.

It is known that the solution of the interpolating problem is a spline from the space

$$
S(T, A)=\{s \in X: \quad<T s, T x>=0 \text { for all } x \in \operatorname{Ker} A\},
$$

corresponding to the operators $T$ and $A$. This spline is called an interpolating spline for a vector $\boldsymbol{r}$.

Under our assumptions the following theorem of existence and uniqueness of an interpolating spline is true.

Theorem 1. ([3], [8]). For each vector $\boldsymbol{r} \in \mathbb{R}^{n}$ there exists an unique interpolating spline.

Concerning splines from the space $S(T, A)$ the following result is known.

Theorem 2. ([3], [8]). An element $s \in X$ is a spline of $S(T, A)$ if and only if there exists a vector $\boldsymbol{\lambda} \in \mathbb{R}^{n}$ such that $T^{*} T s=A^{*} \boldsymbol{\lambda}$.

It allows us to state that an element $s \in X$ belongs to $S(T, A)$ if and only if there exist vectors $\boldsymbol{a} \in \mathbb{R}^{q}$ and $\boldsymbol{\lambda} \in \mathbb{R}^{n}$ such that $s$ can be written as

$$
\begin{aligned}
& s=\sum_{j=1}^{q} a_{j} p_{j}+\sum_{i=1}^{n} \lambda_{i} u_{i}, \\
& \sum_{i=1}^{n} \lambda_{i} k_{i} p_{j}=0, j=1, \ldots, q,
\end{aligned}
$$

where

$$
\begin{aligned}
& q=\operatorname{dimKer} T, p_{1}, \ldots, p_{q} \text { is a basis of } \operatorname{Ker} T \\
& u_{i}=\left(T^{*} T\right)^{-1} A^{*} \boldsymbol{e}_{\boldsymbol{i}}, i=1, \ldots, n, \boldsymbol{e}_{\mathbf{1}}, \ldots, \boldsymbol{e}_{\boldsymbol{n}} \text { is a basis of } \mathbb{R}^{n} .
\end{aligned}
$$

It is known (e.g. [3], [8]) that the solutions of smoothing problems 2 and 3 without the additional condition $\sum_{i=1}^{n} k_{i} g=\sum_{i=1}^{n} r_{i}$ belong to space $S(T, A)$ also. Such splines are called smoothing splines. The main aim of this paper is to investigate the smoothing problems with the additional restriction $\sum_{i=1}^{n} k_{i} g=\sum_{i=1}^{n} r_{i}$.

\section{Analysis of the Smoothing Problem with a Weight}

Let us denote

$$
\begin{aligned}
& Z_{\boldsymbol{r}}=\left\{\boldsymbol{z} \in \mathbb{R}^{n}: \sum_{i=1}^{n} z_{i}=\sum_{i=1}^{n} r_{i}\right\}, \quad X_{\boldsymbol{r}}=A^{-1}\left(Z_{\boldsymbol{r}}\right), \\
& Z_{\boldsymbol{r}, \delta}=\left\{\boldsymbol{z} \in \mathbb{R}^{n}: \sum_{i=1}^{n}\left(z_{i}-r_{i}\right)^{2} \leq \delta\right\}, \quad X_{\boldsymbol{r}, \delta}=A^{-1}\left(Z_{\boldsymbol{r}, \delta}\right) .
\end{aligned}
$$


Theorem 3. There exists a unique solution of Problem 2. An element $s \in X_{\boldsymbol{r}}$ is a solution of this problem if and only if there is a number $\gamma \in \mathbb{R}$ such that

$$
T^{*} T s=A^{*}\left(\gamma \boldsymbol{e}+\frac{1}{\omega}(\boldsymbol{r}-A s)\right),
$$

where $\boldsymbol{e}=(1, \ldots, 1) \in \mathbb{R}^{n}$.

Proof. Taking the pair of operators $T$ and $A$ we introduce the operator $L$

$$
L g=(T g, A g), g \in X
$$

Its values are in the space $V=Y \times \mathbb{R}^{n}$ and the scalar product is defined as

$$
<\left(y^{1}, \boldsymbol{z}^{1}\right),\left(y^{2}, \boldsymbol{z}^{2}\right)>_{V}=<y^{1}, y^{2}>_{Y}+\frac{1}{\omega}<\boldsymbol{z}^{1}, \boldsymbol{z}^{2}>_{\mathbb{R}^{n}} .
$$

We introduce the vector $\mathbf{w}=(0, \boldsymbol{r}) \in V$ and rewrite Problem 2 in the form

$$
\|\| L g-\mathbf{w}\|\|_{V} \longrightarrow \min _{g \in X \boldsymbol{r}} .
$$

By using the interpolating spline $s \boldsymbol{r}$ for the vector $\boldsymbol{r}$ we rewrite

$$
X_{\boldsymbol{r}}=X_{0}+s \boldsymbol{r},
$$

and therefore

$$
L\left(X_{\boldsymbol{r}}\right)=L\left(X_{0}\right)+\mathbf{w}_{\mathbf{r}},
$$

where $\mathbf{w}_{\mathbf{r}}=L(s \boldsymbol{r})=(T s \boldsymbol{r}, \boldsymbol{r})$. To prove the existence of a unique solution of Problem 2 we show that $L\left(X_{0}\right)$ is closed. It is clear that $Z_{0}$ is a closed subspace of $\mathbb{R}^{n}$, which implies that $X_{0}=A^{-1}\left(Z_{0}\right)$ is closed too. The closedness of $L\left(X_{0}\right)$ for a closed subspace $X_{0} \subset X$ is proved in [5], p.13.

Now an element $s \in X_{\boldsymbol{r}}$ is an element with the minimal norm \|\|$L s-\mathbf{w}\|\|_{V}$ (i.e. a solution of Problem 2) if and only if

$$
L s-\mathbf{w} \perp L g \text { for any } g \in X_{0} .
$$

This means that

$$
L^{*}(L s-\mathbf{w}) \perp X_{0} \quad \text { or } \quad L^{*}(L s-\mathbf{w}) \perp \operatorname{Ker} B,
$$

where

$$
B g=\sum_{i=1}^{n} k_{i} g \quad \text { for any } g \in X .
$$

Since $(\operatorname{Ker} B)^{\perp}=\operatorname{Im}\left(B^{*}\right)$ there exists a number $\gamma \in \mathbb{R}$ such that

$$
L^{*}(L s-\mathbf{w})=B^{*} \gamma
$$

The conjugate operator $L^{*}$ can be written as

$$
L^{*} v=T^{*} y+\frac{1}{\omega} A^{*} \boldsymbol{z}, \quad v=(y, \boldsymbol{z}) \in V .
$$


Taking into account also that $B^{*} \gamma=\gamma A^{*} \boldsymbol{e}$, we obtain the equivalent form of the equality (3.1)

$$
T^{*} T s+\frac{1}{\omega} A^{*}(A s-\boldsymbol{r})=\gamma A^{*} \boldsymbol{e},
$$

which proves the theorem.

Corollary 1. The unique solution of Problem 2 is a spline $s \in S(T, A)$, which satisfies the conditions

$$
\begin{aligned}
& T^{*} T s=A^{*} \boldsymbol{\lambda}, \\
& \omega\left(\boldsymbol{\lambda}-\frac{1}{n}<\boldsymbol{\lambda}, \boldsymbol{e}>\boldsymbol{e}\right)+A s=\boldsymbol{r} .
\end{aligned}
$$

Proof. According to Theorem 3 there exists the vector

$$
\boldsymbol{\lambda}=\gamma \boldsymbol{e}+\frac{1}{\omega}(\boldsymbol{r}-A s),
$$

such that $T^{*} T s=A^{*} \boldsymbol{\lambda}$. This means that the solution $s$ belongs to the space $S(T, A)$ (see Theorem 2). Now taking into account the condition

$$
<A s, \boldsymbol{e}>=<\boldsymbol{r}, \boldsymbol{e}>
$$

we get the equality

$$
<\boldsymbol{\lambda}, \boldsymbol{e}>=\gamma n \text {. }
$$

We put this $\gamma$ into $\boldsymbol{\lambda}=\gamma \boldsymbol{e}+\frac{1}{\omega}(\boldsymbol{r}-A s)$, and obtain (3.2).

\section{Equivalence of Two Smoothing Problems}

We denote

$$
\varphi(\omega)=\|A s(\omega)-\boldsymbol{r}\|^{2},
$$

where $s(\omega)$ is the solution of Problem 2.

Theorem 4. If the parameters $\omega$ and $\delta$ are connected by the equation

$$
\varphi(\omega)=\delta,
$$

then the spline $s(\omega)$, i.e. the solution of Problem 2, gives the unique solution of Problem 3.

Proof. Let $\sigma$ be a solution of Problem 3. Note that the spline $s(\omega)$ satisfies also the condition $s(\omega) \in X_{\boldsymbol{r}, \delta} \cap X_{\boldsymbol{r}}$. Therefore

$$
\|T \sigma\| \leq\|T s(\omega)\|
$$

Let us compare the values

$$
\|T \sigma\|^{2}+\frac{1}{\omega}\|A \sigma-r\|^{2} \leq\|T s(\omega)\|^{2}+\frac{1}{\omega} \delta=\|T s(\omega)\|^{2}+\frac{1}{\omega}\|A s(\omega)-\boldsymbol{r}\|^{2} .
$$


This means that $\sigma$ gives a solution of Problem 2 also. Taking into account the uniqueness of the solution of Problem 2, we obtain that $s(\omega)=\sigma$.

Let us suppose $\operatorname{Ker} T \not \subset X_{0}$ and denote

$$
\delta^{*}=\inf \left\{\|A p-\boldsymbol{r}\|^{2}: p \in \operatorname{Ker} T \cap X \boldsymbol{r}\right\} .
$$

This value $\delta^{*}$ will be called the critical value of the smoothing parameter $\delta$. In the case $\delta \geq \delta^{*}$ any $p \in \operatorname{Ker} T \cap X_{\boldsymbol{r}}$ is the solution of Problem 2 .

Now consider the equation $\varphi(\omega)=\delta$ in the case $0<\delta<\delta^{*}$.

Theorem 5. The function $\varphi$ is differentiable, strictly increasing on the interval $(0,+\infty)$ and

$$
\lim _{\omega \rightarrow+0} \varphi(\omega)=0, \quad \lim _{\omega \rightarrow+\infty} \varphi(\omega) \geq \delta^{*} .
$$

Proof. According to (2.1), (2.2) the spline $s(\omega)$ can be written as

$$
\begin{aligned}
& s(\omega)=\sum_{j=1}^{q} a_{j}(\omega) p_{j}+\sum_{i=1}^{n} \lambda_{i}(\omega) u_{i} \\
& \sum_{i=1}^{n} \lambda_{i}(\omega) k_{i} p_{j}=0, \quad j=1, \ldots, q .
\end{aligned}
$$

Its coefficients depend on $\omega$. The coefficients $\boldsymbol{a}(\omega), \boldsymbol{\lambda}(\omega)$ of the spline $s(\omega)$ are differentiable as the solution of the following system of the algebraic equations

$$
\left\{\begin{array}{l}
\omega\left(\lambda_{i}(\omega)-\frac{1}{n} \sum_{l=1}^{n} \lambda_{l}(\omega)\right)+k_{i} s(\omega)=r_{i}, \quad i=1, \ldots, n \\
\sum_{i=1}^{n} \lambda_{i}(\omega) k_{i} p_{j}=0, \quad j=1, \ldots, q
\end{array}\right.
$$

If the coefficients are differentiable with respect to $\omega$, then the function $\varphi$ is continuously differentiable with respect to $\omega$ also. It is easy to see that

$$
\varphi^{\prime}(\omega)=2<A s^{\prime}(\omega), A s(\omega)-r>
$$

By differentiating we obtain that $s^{\prime}(\omega)$ is a spline of $S(T, A)$ with the coefficients $\boldsymbol{a}^{\prime}(\omega), \boldsymbol{\lambda}^{\prime}(\omega)$ :

$$
\begin{aligned}
& s^{\prime}(\omega)=\sum_{j=1}^{q} a_{j}^{\prime}(\omega) p_{j}+\sum_{i=1}^{n} \lambda_{i}^{\prime}(\omega) u_{i}, \\
& \sum_{i=1}^{n} \lambda_{i}^{\prime}(\omega) k_{i} p_{j}=0, \quad j=1, \ldots, q .
\end{aligned}
$$

It means that

$$
T^{*} T s^{\prime}(\omega)=A^{*} \lambda^{\prime}(\omega)
$$

Thus

$$
\left\|T s^{\prime}(\omega)\right\|^{2}=<A s^{\prime}(\omega), \lambda^{\prime}(\omega)>
$$


In order to obtain $\boldsymbol{\lambda}^{\prime}(\omega)$, let us differentiate the system (4.1)

$$
\boldsymbol{\lambda}(\omega)-\gamma(\omega) \boldsymbol{e}+\omega\left(\boldsymbol{\lambda}^{\prime}(\omega)-\gamma^{\prime}(\omega) \boldsymbol{e}\right)+A s^{\prime}(\omega)=0,
$$

where $\gamma(\omega)=\frac{1}{n} \sum_{i=1}^{n} \lambda_{i}(\omega)$. Therefore

$$
\boldsymbol{\lambda}(\omega)-\gamma(\omega) \boldsymbol{e}=-\omega\left(\boldsymbol{\lambda}^{\prime}(\omega)-\gamma^{\prime}(\omega) \boldsymbol{e}\right)-A s^{\prime}(\omega) .
$$

By the equality

$$
\boldsymbol{\lambda}(\omega)-\gamma(\omega) \boldsymbol{e}=\frac{1}{\omega}(\boldsymbol{r}-A s(\omega))
$$

we get

$$
A s(\omega)-\boldsymbol{r}=\omega^{2}\left(\boldsymbol{\lambda}^{\prime}(\omega)-\gamma^{\prime}(\omega) \boldsymbol{e}\right)+\omega A s^{\prime}(\omega) .
$$

Therefore

$$
\varphi^{\prime}(\omega)=2 \omega^{2}<A s^{\prime}(\omega), \boldsymbol{\lambda}^{\prime}(\omega)>-2 \omega^{2}<A s^{\prime}(\omega), \gamma^{\prime}(\omega) \boldsymbol{e}>+2 \omega\left\|A s^{\prime}(\omega)\right\|^{2} .
$$

Now taking into account that

$$
<A s^{\prime}(\omega), \gamma^{\prime}(\omega) \boldsymbol{e}>=\gamma^{\prime}(\omega)(<A s(\omega), \boldsymbol{e}>)^{\prime}=\gamma^{\prime}(\omega)(<\boldsymbol{r}, \boldsymbol{e}>)^{\prime}=0,
$$

we obtain

$$
\varphi^{\prime}(\omega)=2 \omega^{2}\left\|T s^{\prime}(\omega)\right\|^{2}+2 \omega\left\|A s^{\prime}(\omega)\right\|^{2} .
$$

It means that $\varphi^{\prime}(\omega)>0$ for positive $\omega$, and hence the function $\varphi$ is strictly increasing on the interval $(0,+\infty)$.

We denote the interpolating spline for the vector $\boldsymbol{r}$ by $s \boldsymbol{r}$. From

$$
\|T s(\omega)\|^{2}+\frac{1}{\omega}\|A s(\omega)-r\|^{2} \leq\|T s \boldsymbol{r}\|^{2}+\frac{1}{\omega}\|A s \boldsymbol{r}-r\|^{2}=\|T s \boldsymbol{r}\|^{2}
$$

it follows that $\frac{1}{\omega}\|A s(\omega)-r\|^{2}$ is bounded and $\lim _{\omega \rightarrow+0} A s(\omega)=r$. Therefore we get

$$
\lim _{\omega \rightarrow+0} \varphi(\omega)=0 .
$$

To prove the inequality $\lim _{\omega \rightarrow+\infty} \varphi(\omega) \geq \delta^{*}$ it is enough to consider the case, when the function $\varphi(\omega)$ is bounded. If the strictly increasing function function $\varphi(\omega)$ is unbounded, then this inequality holds automatically.

In case of bounded function $\varphi(\omega)$, the functions $k_{i} s(\omega), i=1, \ldots, n$, are limited and if $\omega \rightarrow+\infty$ then from the first equation of the system (4.1) we get

$\lim _{\omega \rightarrow+\infty}\left(\lambda_{i}(\omega)-\frac{1}{n} \sum_{j=1}^{n} \lambda_{j}(\omega)\right)=0, \quad$ or $\quad \lim _{\omega \rightarrow+\infty}\left(\lambda_{i}(\omega)-\gamma(\omega)\right)=0, i=1, \ldots, n$.

Therefore we can write

$$
\lambda_{i}(\omega)=\gamma(\omega)+\beta_{i}(\omega), i=1, \ldots, n,
$$


where $\lim _{\omega \rightarrow+\infty} \beta_{i}(\omega)=0$. So from the second equation of (4.1) we have

$$
\lim _{\omega \rightarrow+\infty} \sum_{i=1}^{n}\left(\gamma(\omega)+\beta_{i}(\omega)\right) k_{i} p_{j}=0, \quad j=1, \ldots, q .
$$

The conditions $\lim _{\omega \rightarrow+\infty} \beta_{i}(\omega)=0, i=1, \ldots, n$ imply

$$
\lim _{\omega \rightarrow+\infty} \gamma(\omega) \sum_{i=1}^{n} k_{i} p_{j}=0, j=1, \ldots, q .
$$

Taking into account that $\operatorname{Ker} T \not \subset X_{0}$ we get $\lim _{\omega \rightarrow+\infty} \gamma(\omega)=0$. It means that for all coefficients $\lambda_{i}(\omega), i=1, \ldots, n$,

$$
\lim _{\omega \rightarrow+\infty} \lambda_{i}(\omega)=0
$$

To prove the inequality

$$
\lim _{\omega \rightarrow+\infty} \varphi(\omega) \geq \delta^{*}
$$

we will show that there exists such a sequence of natural numbers $\left(\omega_{m}\right)_{m \in \mathbb{N}}$ that

$$
\lim _{m \rightarrow+\infty} \omega_{m}=+\infty \text { and } \lim _{m \rightarrow+\infty} \boldsymbol{a}\left(\omega_{m}\right) \in \mathbb{R}^{q}
$$

Let us denote $\boldsymbol{a}^{*}=\lim _{m \rightarrow+\infty} \boldsymbol{a}\left(\omega_{m}\right)$ and consider $p^{*}=\sum_{j=1}^{q} a_{j}^{*} p_{j}$. It is easy to see that $p^{*} \in X_{\boldsymbol{r}}$ because of

$$
\begin{aligned}
\sum_{i=1}^{n} k_{i} \sum_{j=1}^{q} a_{j}^{*} p_{j} & =\lim _{m \rightarrow+\infty} \sum_{i=1}^{n} k_{i} \sum_{j=1}^{q} a_{j}\left(\omega_{m}\right) p_{j} \\
& =\lim _{m \rightarrow+\infty}\left(\sum_{i=1}^{n} k_{i} s\left(\omega_{m}\right)-\sum_{i=1}^{n} k_{i} \sum_{j=1}^{n} \lambda_{i}\left(\omega_{m}\right) u_{j}\right)=\sum_{i=1}^{n} r_{i} .
\end{aligned}
$$

Therefore

$$
\lim _{m \rightarrow+\infty} \varphi\left(\omega_{m}\right)=\left\|A p^{*}-\boldsymbol{r}\right\|^{2} \geq \delta^{*}
$$

To prove the existence of sequence (4.3) we will show that sequence $(\boldsymbol{a}(m))_{m \in \mathbb{N}}$ is bounded (in this case it has a convergent subsequence). Taking into account that $k_{i} s(m), i=1, \ldots, n$ are bounded, we obtain that

$$
k_{i}\left(\sum_{j=1}^{q} a_{j}(m) p_{j}\right)=\sum_{j=1}^{q} a_{j}(m) k_{i} p_{j}, \quad i=1, \ldots, n
$$

are bounded too. By using the matrix $V=\left(k_{i} p_{j}\right)_{i=1, \ldots, n, j=1, \ldots, q}$ we rewrite the vector with the coordinates $(4.4)$ as $V \boldsymbol{a}(\mathrm{m})$. The boundedness of $V \boldsymbol{a}(\mathrm{m})$ implies the boundedness of $V^{T} V \boldsymbol{a}(m)$. To prove the boundedness of the vector $\boldsymbol{a}(m)$ it is enough to note that the matrix $V^{T} V$ is invertible as Gram's matrix, which corresponds to the linearly independent vectors $A p_{j}, j=1, \ldots, q$. These vectors are linearly independent because of $\operatorname{Ker} T \cap \operatorname{Ker} A=\{0\}$. 
Corollary 2. For $\delta \in\left(0, \delta^{*}\right)$ the connection equation $\varphi(\omega)=\delta$ has a unique solution.

Corollary 3. For any values $\delta \in(0,+\infty)$ Problem 3 has the solution and this solution is the spline from the space $S(T, A)$.

\section{References}

[1] S. Asmuss and N. Budkina. Splines in convex sets under constraints of twosided inequality type in a hiperplane. Math. Model. Anal., 13(4):461-470, 2008. (doi:10.3846/1392-6292.2008.13.461-470)

[2] L.I. Boneva, D.G. Kendall and I. Stefanov. Spline transformations: Three new diagnostic aids for statistical data - analyst. J. Royal Statist. Soc. Ser. B, 33:170, 1971.

[3] R. J. Carroll, P. Hall, T. V. Apanasovich and X. Lin. Histospline method in nonparametric regression models with application to clustered/longitudinal data. Statistica Sinica, 14:649-674, 2004.

[4] P. van Casteren. The frequency polygon reconsidered. Computational Statistics and Data Analysis, 24(1):1-8, 1997.

[5] P. Costantini and F. Pelosi. Shape preserving histogram approximation. Advances in Computational Mathematics, 26(1-3):205-230, 2007.

[6] D. Docenko and K. Berzins. Spline histogram method for reconstruction of probability density functions of clusters of galaxies. In G. Contopoulos N. Voglis (Eds.)(Ed.), Galaxies and Chaos, pp. 294-301. Springer-Verlag Berlin Heidelberg, 2003.

[7] M. Fischer and P. Oja. Convergence rate of rational spline histopolation. Math. Model. Anal., 12(1):29-38, 2007. (doi:10.3846/1392-6292.2007.12.29-38)

[8] Scott N. Kersey. On the problems of smoothing and near-interpolation. Math. Comput., 72(244):1873-1885, 2003.

[9] P.-J. Laurent. Approximation et optimisation. Hermann, Paris, 1972.

[10] I.J. Schoenberg. Splines and histograms. Spline functions and approximation theory. In Proc. Sympos. Univ. Alberta, Edmonton, Alta, pp. 277-327, 1972.

[11] V. Vershinin, Yu.Zavyalov and N. Pavlov. Extremal properties of splines and the smoothing problem. Nauka, Novosibirsk, 1988. (in Russian)

[12] G. Wahba. Spline Models for Observational Data. SIAM, Philadelphia, 1991.

[13] H. Wang. A rational spline model approximation and control of output probability density functions for dynamic stochastic systems. Transactions of the Institute of Measurement and Control, 25(2):93-105, 2003. 